\title{
Complete Bilateral Teleoperation System for a Rotorcraft UAV with Time-Varying Delay
}

\author{
Lucio R. Salinas, ${ }^{1}$ Emanuel Slawiñski, ${ }^{2}$ and Vicente A. Mut $^{2}$ \\ ${ }^{1}$ Centro Avanzado de Ingeniería Eléctrica y Electrónica (AC3E), Universidad Técnica Federico Santa María, \\ Avenida España 1680, 2390123 Valparaíso, Chile \\ ${ }^{2}$ Instituto de Automática (INAUT), Universidad Nacional de San Juan, CONICET, Avenida San Martín Oeste 1109, \\ J5400ARL San Juan, Argentina \\ Correspondence should be addressed to Lucio R. Salinas; lucio.salinas@usm.cl
}

Received 17 February 2015; Revised 9 July 2015; Accepted 16 July 2015

Academic Editor: Giuseppe Rega

Copyright ( $\odot 2015$ Lucio R. Salinas et al. This is an open access article distributed under the Creative Commons Attribution License, which permits unrestricted use, distribution, and reproduction in any medium, provided the original work is properly cited.

This paper proposes the use of simple proportional plus damping injection $(\mathrm{P}+\mathrm{d})$ controllers for delayed bilateral teleoperation of a rotorcraft UAV. The proposed control scheme involves $\mathrm{P}+\mathrm{d}$ remote and local controllers, considers master and slave dynamics, and takes into account asymmetric time-varying delays. The stability of the proposed teleoperation system is analyzed using Lyapunov-Krasovskii functionals and delay-dependent stability criteria are obtained under linear-matrix-inequalities conditions. The performance of the teleoperation scheme is tested driving a virtual nonlinear 6DOF dynamic model of a minihelicopter in a human-in-the-loop simulation.

\section{Introduction}

Over the last two decades, unmanned aerial vehicles (UAVs) have become an important scientific research topic. The reason for this is based on three main aspects: UAVs avoid the human risk inherent to manned aerial vehicles in many situations (e.g., hostile environment and adverse weather condition); they are generally far less expensive and smaller than their manned counterparts; and they can reach places and/or overfly specific areas that other vehicles cannot.

Within UAVs, rotorcraft-based UAVs (RUAVs) have unique flight capabilities such as hover, vertical take-off and landing, and side-slip, which cannot be achieved by conventional fixed-wing aircraft. The hovering mode and flying at low speed make RUAVs ideal for reconnaissance missions and very useful in rescue missions [1]. These singular features called the attention of many academic research groups in the last years. Although there has been much research in the area of RUAVs, the majority of published works have covered the design and implementation of control systems for autonomous navigation only (see [2] and references therein).
However, a lot of inspection, surveillance, and monitoring tasks cannot be easily performed with a fully autonomous vehicle; the need for high-level reasoning and specific knowledge inherent in the task make RUAV teleoperation more suitable for these missions [3].

In general, UAV teleoperation systems have three elements: a ground station (local site), where a human operator receives information and drives a hand-controller device (master device); a UAV (slave vehicle or device) that receives control signals and flies through an environment (remote site); and a communication channel that links both sites. The master device at the local site and the slave vehicle at the remote site are endowed with local controllers as seen in Figure 1 . The information provided in the local site is usually visual and the operator lacks other sensory information that a pilot of a manned aircraft would normally perceive, such as sound, vibration, and motion [4]. Additionally, the communication channel adds delays in the range of 100 to 1670 milliseconds (and even more) [5] that can produce instability or poor performance $[6,7]$.

In recent years, several authors have developed teleoperation control systems for RUAVs; even so, only a few have 


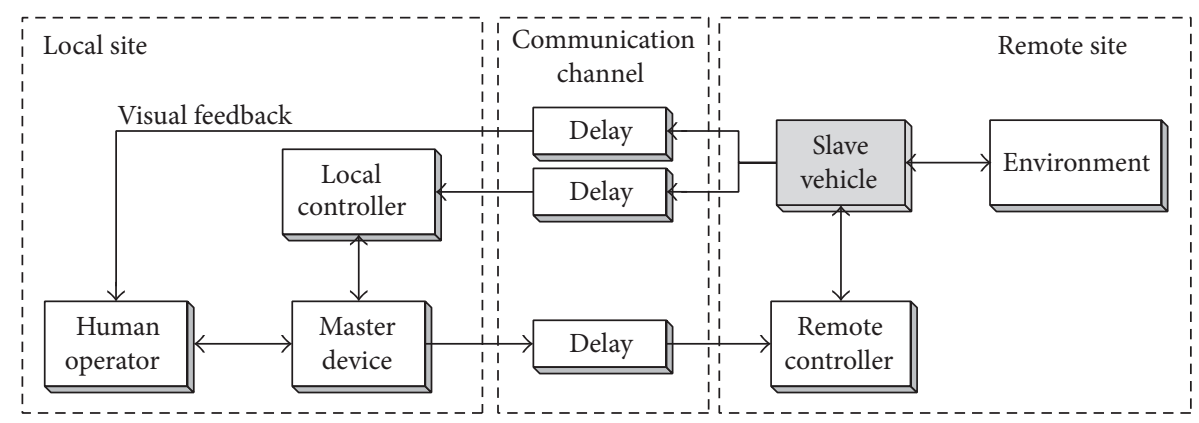

FIGURE 1: Block diagram of a UAV teleoperation system.

focused on delayed bilateral teleoperation schemes. Bilateral teleoperation provides the human operator with a haptic feedback; this signal can complement the visual information to improve the telepresence and enhance safety; as a drawback, it can destabilize the closed-loop teleoperation system $[8,9]$.

In [10] the influence of different force-feedback settings on a human operator is studied and the wave variables conventional teleoperation technique $[11,12]$ is used to reduce the negative effects of time delays. In [13] a bilateral teleoperation of multiple mobile slave agents coupled to a single master robot under constant communication delays is achieved using a passifying PD-based controller; nevertheless, it addresses only the master-slave position-position teleoperation and thus it is not suitable for UAVs with unbounded workspace. In [14-17] a bilateral teleoperation of multiple UAVs is performed by a single human operator and the stability is analyzed employing passivity theory. In the first two researches, the passivity of the master side is obtained applying feedback r-passivity notion [18]; then, it is claimed that the passivity of the teleoperation system can be easily enforced using any of the techniques developed in conventional teleoperation settings. In the latter two investigations, passive set-position modulation (PSPM) framework [19] is utilized to theoretically guarantee masterpassivity/VPs-stability of the closed-loop teleoperation system (where VPs refer to the virtual points followed by each UAV). In [20] the port-based modeling network, in particular, port-Hamiltonian systems and bond graphs, is used in the bilateral teleoperation of aerial robots, where a variable impedance master controller and a virtual slave system allow the design of a passive teleoperation control architecture.

Contribution. In bilateral teleoperation of manipulator robots, proportional plus damping injection $(\mathrm{P}+\mathrm{d})$ controllers have gained momentum in the last few years [2123]. Due to its simplicity and robustness, this work proposes the use of $\mathrm{P}+\mathrm{d}$ controllers for bilateral teleoperation of a RUAV with time-varying delay considering the master device and slave vehicle dynamics. The main contribution of the paper involves combining in a simple way $\mathrm{P}+\mathrm{d}$ controllers, a trajectory tracking controller, an impedance control based on fictitious force, an elastic coupling, and a cascade PID architecture, to achieve a stable bilateral teleoperation of an underactuated 6DOF minihelicopter in presence of timevarying delay. The stability analysis carried out gives as a result a delay-dependent LMI condition, which can be held if the control parameters are set from such condition, assuring thus a stable performance. In the state of the art in the field of delayed bilateral teleoperation of aerial vehicles, most controllers are based on passivity theory and they generally include a difficult calibration procedure. Different from this, the proposed scheme involves a simple parameters setting where the control blocks $(\mathrm{P}+\mathrm{d}$ control, trajectory tracking controller, impedance control, etc.) can be set independently in practice.

To test the performance of the teleoperation scheme a human-in-the-loop (HITL) simulation is carried out; there, a human operator drives a RUAV through an outdoor environment and has visual and force feedback under a communication channel with asymmetric time-varying delay. Two master devices are used increasing the maneuverability of the RUAV and the visual feedback is only generated by an onboard camera providing a field of view like the one achievable in practice.

The rest of the paper is organized as follows. Sections 2 and 3 introduce the models of the master device and slave vehicle, respectively. Section 4 presents some mathematical properties and assumptions. The $\mathrm{P}+\mathrm{d}$ controllers are proposed and explained in Section 5. In Section 6 the stability analysis is outlined. The complete teleoperation system is presented in Section 7. The HITL simulation framework and the simulation results are provided in Section 8. Finally, the conclusions are given in Section 9.

\section{Master Device}

The master device at the local site is composed of two parts: the translational master and the rotational master. The first is a 3-degree-of-freedom (DOF) device with force feedback which controls the translation rates of the UAV and the second is a $1 \mathrm{DOF}$ device (a two-pedal configuration) which commands the rotation rate of the UAV (see Figure 2).

2.1. Translational Master Device. The typical nonlinear dynamic model in Cartesian coordinates is used to represent the 3DOF device:

$$
\mathbf{M}(\mathbf{x}) \ddot{\mathbf{x}}+\mathbf{C}(\mathbf{x}, \dot{\mathbf{x}}) \dot{\mathbf{x}}+\mathbf{g}_{\mathrm{m}}(\mathbf{x})=\mathbf{f}_{\mathbf{m}}^{*}+\mathbf{f}_{\mathbf{h}},
$$




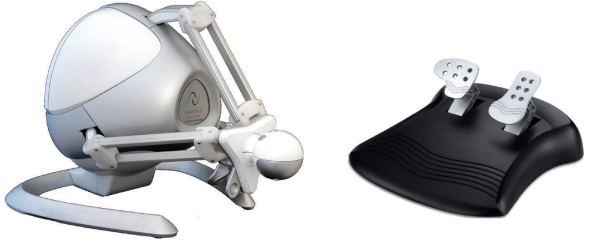

Figure 2: 3DOF Novint Falcon translational master device and 1DOF Genius rotational master device.

where $\mathbf{x} \in \mathfrak{R}^{3}$ is the end-effector position, $\mathbf{M} \in \mathfrak{R}^{3 \times 3}$ is the inertia matrix, $\mathbf{C} \in \mathfrak{R}^{3 \times 3}$ is the matrix representing centripetal and Coriolis effects, $\mathbf{g}_{\mathrm{m}} \in \mathfrak{R}^{3}$ is the gravitational force, and $\mathbf{f}_{\mathrm{m}}^{*} \in \mathfrak{R}^{3}$ and $\mathbf{f}_{\mathrm{h}} \in \mathfrak{R}^{3}$ are the control and human operator forces applied to the translational master, respectively.

The gravitational force is precompensated by the controller $\mathbf{f}_{\mathrm{m}}^{*}$; that is, $\mathbf{f}_{\mathrm{m}}^{*}=\mathbf{f}_{\mathrm{m}}+\mathbf{g}_{\mathrm{m}}(\mathbf{x})$. The final model for the translational master is the following:

$$
\mathbf{M}(\mathbf{x}) \ddot{\mathbf{x}}+\mathbf{C}(\mathbf{x}, \dot{\mathbf{x}}) \dot{\mathbf{x}}=\mathbf{f}_{\mathbf{m}}+\mathbf{f}_{\mathbf{h}} .
$$

2.2. Rotational Master Device. The $1 \mathrm{DOF}$ device is modeled as two forced mass-spring-damper systems:

$$
m_{r} \ddot{\mathbf{q}}+b_{r} \dot{\mathbf{q}}+k_{r} \mathbf{q}=\boldsymbol{\tau}_{\mathbf{h}},
$$

where $\mathbf{q}=\left[\begin{array}{ll}q_{\mathrm{rp}} & q_{\mathrm{lp}}\end{array}\right]^{\mathrm{T}} \in \mathfrak{R}^{2}$ is a vector indicating the joint angular displacement of the right and left pedals; $m_{r}, b_{r}$, and $k_{r}$ are the pedals inertia, viscosity, and stiffness, respectively; and $\tau_{\mathbf{h}}=\left[\begin{array}{ll}\tau_{\mathrm{rf}} & \tau_{\mathrm{lf}}\end{array}\right]^{\mathrm{T}} \in \mathfrak{R}^{2}$ is a vector that indicates the human operator's right and left feet torques.

The output signal $q_{p}$ of the rotational master is the difference between the joint angular displacements of the pedals; that is, $q_{p}=q_{\mathrm{rp}}-q_{\mathrm{lp}}$.

\section{Slave Vehicle}

RUAVs belong to the class of underactuated mechanical systems, which have fewer control inputs than degrees of freedom. The $6 \mathrm{DOF}$ dynamic model of a RUAV is very complex, and it is certainly difficult to obtain the mathematical equations and parameters that follow its physical model [24]. One practical solution to this problem could be to use a more data-driven/model-free design framework of the control scheme (see [25] and references therein). Another solution, applied in this work, is to use a simplified fully actuated $4 \mathrm{DOF}$ model in the controller design stage and then couple it to a full RUAV model. In Section 8, the interaction between the $4 \mathrm{DOF}$ model and a more realistic underactuated 6DOF RUAV model of a minihelicopter is explained.

In Figure 3, the reference point $o_{0}$ on the surface of the earth is the origin of a right handed orthogonal system of axes $\left(o_{0} x_{0} y_{0} z_{0}\right), o_{0} x_{0}$ points to the north, $o_{0} y_{0}$ points to the east, and $o_{0} z_{0}$ points vertically "down" along the gravity vector (NED Cartesian system). The kinematics and dynamics of the
4DOF vehicle are equivalent to the ones of a rigid body whose roll and pitch movements are constrained; hence, its body axes $\left(o_{E} x_{E} y_{E} z_{E}\right)$ are tantamount to a Cartesian coordinate system "tied" to the earth by means of gravity vector with the difference being that the origin $o_{E}$ coincides with the center of gravity of the aircraft and $o_{E} x_{E}$ axis points forward along the vehicle.

The fully actuated $4 \mathrm{DOF}$ dynamic model is the following:

$$
\mathrm{D} \dot{\eta}^{*}+\mathbf{Q}\left(\boldsymbol{\eta}^{*}\right) \boldsymbol{\eta}^{*}+\mathrm{g}_{\mathrm{s}}=\sigma_{\mathrm{s}}^{*}+\sigma_{\mathrm{e}}
$$

where $\boldsymbol{\eta}^{*}=\left[\begin{array}{llll}v_{x_{E}}^{r} & v_{y_{E}}^{r} & v_{z_{E}}^{r} & \omega_{z_{E}}^{r}\end{array}\right]^{\mathrm{T}} \in \mathfrak{R}^{4}$ is the RUAV velocity vector with $v_{x_{E}}^{r}, v_{y_{E}}^{r}$, and $v_{z_{E}}^{r}$ representing the forwardbackward, lateral, and downward-upward linear velocity, respectively, and $\omega_{z_{E}}^{r}$ is the angular velocity as seen in Figure 3. $\sigma_{\mathrm{e}} \in \mathfrak{R}^{4}$ is the force/torque caused by the interaction between the environment and the RUAV and $\boldsymbol{\sigma}_{\mathrm{s}}^{*} \in$ $\mathfrak{R}^{4}$ is the control force/torque applied to the RUAV.

The inertia matrix $\mathbf{D} \in \mathfrak{R}^{4 \times 4}$, the Coriolis matrix $\mathbf{Q}\left(\boldsymbol{\eta}^{*}\right) \epsilon$ $\mathfrak{R}^{4 \times 4}$, and the gravity vector $\mathbf{g}_{\mathrm{s}} \in \mathfrak{R}^{4}$ are the following:

$$
\begin{aligned}
& \mathbf{D}=\left[\begin{array}{cccc}
m & 0 & 0 & 0 \\
0 & m & 0 & 0 \\
0 & 0 & m & 0 \\
0 & 0 & 0 & i_{r}
\end{array}\right], \\
& \mathbf{Q}=\left[\begin{array}{cccc}
0 & -m \omega_{z_{E}}^{r} & 0 & 0 \\
m \omega_{z_{E}}^{r} & 0 & 0 & 0 \\
0 & 0 & 0 & 0 \\
0 & 0 & 0 & 0
\end{array}\right], \\
& \mathbf{g}_{\mathbf{s}}=\left[\begin{array}{llll}
0 & 0 & -m g & 0
\end{array}\right]^{\mathrm{T}},
\end{aligned}
$$

where $m, i_{r}$, and $g$ are positive constants denoting the mass, the rotational inertia, and the gravitational acceleration of the RUAV, respectively.

To devise the translational and rotational control actions independently the RUAV dynamic model is decoupled. For this purpose, the control force/torque $\sigma_{\mathrm{s}}^{*}$ in (4) is established as follows:

$$
\sigma_{\mathrm{s}}^{*}=\sigma_{\mathrm{s}}+\mathbf{Q}\left(\eta^{*}\right) \eta^{*}+\mathrm{g}_{\mathrm{s}}
$$

Including (6) in (4) we obtain $\mathbf{D} \dot{\eta}^{*}=\sigma_{\mathrm{s}}+\boldsymbol{\sigma}_{\mathrm{e}}$, being the translational slave dynamics

$$
m \dot{\boldsymbol{\eta}}=\mathbf{f}_{\mathrm{s}}+\mathbf{f}_{\mathrm{e}}
$$

and the rotational slave dynamics

$$
i_{r} \dot{\eta}_{r}=\tau_{s}+\tau_{e}
$$

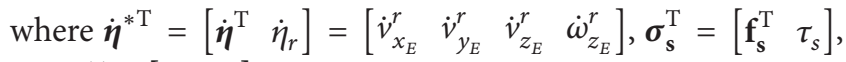
and $\boldsymbol{\sigma}_{\mathrm{e}}^{\mathrm{T}}=\left[\begin{array}{ll}\mathbf{f}_{\mathbf{e}}^{\mathrm{T}} & \tau_{e}\end{array}\right]$. 


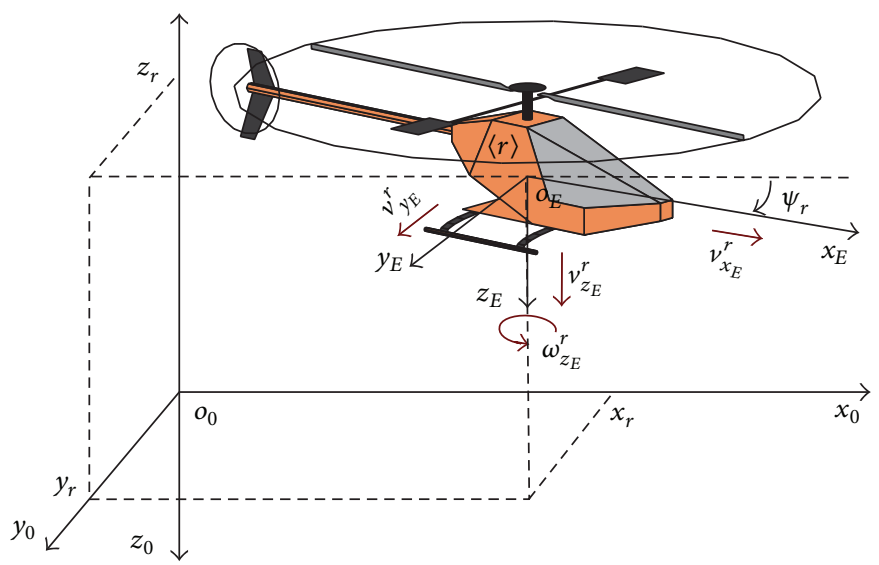

Figure 3: Fully actuated 4DOF RUAV.

\section{Preliminaries}

The following properties, assumptions, and lemma will be used in this paper.

Property 1. The inertia matrix $\mathbf{M}$ is a symmetric positivedefinite function and there exist positive constants $m_{1}$ and $m_{2}$ such that $m_{1} \mathbf{I} \leq \mathbf{M} \leq m_{2} \mathbf{I}$.

Property 2. The matrix $\dot{\mathbf{M}}-2 \mathbf{C}$ is skew symmetric.

Property 3. There exists a positive constant $k_{c}$ such that $\mathbf{C} \dot{\mathbf{x}} \leq$ $k_{c}|\dot{\mathbf{x}}|$.

Assumption 1. The human operator and the environment behave in a nonpassive way and they are represented by the following models:

$$
\begin{aligned}
& \mathbf{f}_{\mathbf{h}}=-m_{h} \ddot{\mathbf{x}}-\alpha_{h} \dot{\mathbf{x}}+\mathbf{f}_{\mathbf{h}}^{*} \\
& \mathbf{f}_{\mathbf{e}}=-m_{e} \dot{\boldsymbol{\eta}}-\alpha_{e} \boldsymbol{\eta}+\mathbf{f}_{\mathbf{e}}^{*},
\end{aligned}
$$

where $m_{i}, \alpha_{i}$, and $\mathbf{f}_{\mathbf{i}}^{*}$ with $i=h, e$ are the inertia, damping, and exogenous force of the human operator and environment, respectively. The exogenous forces are subject to $\left\|\mathbf{f}_{\mathbf{i}}^{*}\right\|_{\infty}<$ $\bar{f}_{i}^{*}<+\infty$, and $\bar{f}_{i}^{*}$ is a positive constant.

Note. Models (9) and (10) are modified versions of the secondorder LTI models successfully used by other researchers [2628]. In (9) and (10), the stiffness of the human operator and environment is ignored considering the operator does not want to maintain a certain hand position and the vehicle makes no physical contact with the environment. Also, $m_{i}$ and $\alpha_{i}$ in (9) and (10) were taken as scalars but they can be diagonal matrices or, more generally, positive-definite matrices.

Assumption 2. The communication channel is represented by a forward time-varying delay $h_{1}(t)$ (local site $\rightarrow$ remote site) and a backward time-varying delay $h_{2}(t)$ (remote site $\rightarrow$ local site). These time delays are considered bounded; that is, there exist positive scalars $\bar{h}_{1}$ and $\bar{h}_{2}$ such that $0 \leq h_{1}(t) \leq \bar{h}_{1}$ and $0 \leq h_{2}(t) \leq \bar{h}_{2}$ for all $t$.

Lemma 3 (see [22]). For vector functions $\mathbf{a}(\cdot)$ and $\mathbf{b}(\cdot), a$ positive-definite matrix $\Delta$, and a time-varying scalar $h(t)$ with $0 \leq h(t) \leq \bar{h}$, the following inequality holds:

$$
\begin{array}{r}
-2 \mathbf{a}^{\mathrm{T}}(t) \int_{t-h(t)}^{t} \mathbf{b}(\xi) \mathrm{d} \xi-\int_{t-h(t)}^{t} \mathbf{b}^{\mathrm{T}}(\xi) \Delta \mathbf{b}(\xi) \mathrm{d} \xi \\
\leq h(t) \mathbf{a}^{\mathrm{T}}(t) \Delta^{-1} \mathbf{a}(t) \leq \bar{h}(t) \mathbf{a}^{\mathrm{T}}(t) \Delta^{-1} \mathbf{a}(t) .
\end{array}
$$

\section{P+d Controllers for Bilateral Teleoperation}

The teleoperation system has two controllers (Figure 1) that interact with the master device and consequently the human operator on the one hand and with the slave vehicle and its environment on the other hand. Both controllers have a simple $\mathrm{P}+\mathrm{d}$ structure; thus they possess few parameters that can be quickly calibrated.

5.1. Remote Controller. The aim of the remote controller is to synchronize the slave vehicle's linear and angular velocities $\left(\boldsymbol{\eta}, \eta_{r}\right)$ with the position and angular displacement of the master device, respectively. The control actions are the following:

$$
\begin{aligned}
& \mathbf{f}_{\mathbf{s}}=k_{s}\left(k_{n} \mathbf{x}\left(t-h_{1}\right)-\boldsymbol{\eta}\right)-\alpha_{s} \mathbf{z}, \\
& \tau_{s}=k_{\mathrm{sr}}\left(k_{\mathrm{nr}} q_{p}\left(t-h_{1}\right)-\eta_{r}\right)-\alpha_{\mathrm{sr}} z_{r},
\end{aligned}
$$

where $k_{s}, k_{\mathrm{sr}}, \alpha_{s}$, and $\alpha_{\mathrm{sr}}$ are positive constant parameters representing the proportional gain and acceleration-dependent damping added by the $\mathrm{P}+\mathrm{d}$ controllers. $k_{n}$ and $k_{\mathrm{nr}}$ are positive constant parameters that map master position and angular displacement to velocity commands and $\mathbf{z}^{*} \in \mathfrak{R}^{4}$ is an auxiliary vector defined as

$$
\mathbf{z}^{*}+\gamma \dot{\mathbf{z}}^{*}=\dot{\boldsymbol{\eta}}^{*}
$$


where $\gamma \rightarrow 0^{+}$and $\mathbf{z}^{* \mathrm{~T}}=\left[\begin{array}{ll}\mathbf{z}^{\mathrm{T}} & z_{r}\end{array}\right]$. That is, $\mathbf{z}^{*}$ represents the RUAV acceleration $\dot{\boldsymbol{\eta}}^{*}$ at an infinitesimal time instant before $t$.

The end-effector position vector of the translational master and the angular displacement of the rotational master generate linear and angular velocity setpoints for the remote controller. This relation between master device and slave vehicle helps overcome the master-slave kinematic dissimilarity typical of mobile robot teleoperation; that is, the master device has bounded workspace and the slave vehicle has unbounded workspace.

5.2. Local Controller. The objective of the local controller is to provide the human operator with a haptic cue that represents the mismatch between the commanded velocity (specified by the end-effector position vector) and the velocity of the slave. The mismatch gives different kind of information: dynamics of the slave vehicle, external disturbances on the RUAV motion, and quality of velocity tracking in the remote site. The local controller only affects the translational master device as the rotational one is unactuated. The control action is the following:

$$
\mathbf{f}_{\mathbf{m}}=-k_{m}\left(\mathbf{x}-k_{n}^{-1} \boldsymbol{\eta}\left(t-h_{2}\right)\right)-\alpha_{m} \dot{\mathbf{x}}
$$

where $k_{m}$ and $\alpha_{m}$ are positive constant parameters representing a relative spring depending on the mismatch between the master reference and the RUAV linear velocity and damping added by the $\mathrm{P}+\mathrm{d}$ controller, respectively. $k_{n}^{-1}$ maps slave vehicle's velocity to master position.

\section{Stability of the Bilateral Teleoperation System}

The rotational part of the teleoperation system is unilateral; thus the complexity of its control is much reduced; therefore, only the stability of the translational part is analyzed using the theory of Lyapunov-Krasovskii [29]. It is important to remark that there is not an equilibrium point but a Krasovskiilike equilibrium solution that depends on the state $\phi^{\mathrm{T}}=$

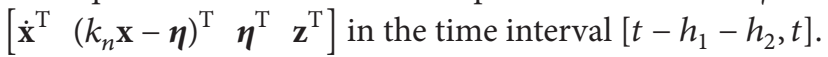

Theorem 4. Consider a delayed bilateral teleoperation system where a human operator (9) using a master device (2) drives a RUAV (7) interacting with an environment (10), and control laws (12), (13), and (15) are included. For positive constant parameters $k_{m}, k_{s}, k_{n}, \alpha_{m}$, and $\alpha_{s}$, slave vehicle mass $m$, and maximum time delays $\bar{h}_{1}$ and $\bar{h}_{2}$, considering Assumptions 1 and 2 and Properties 1, 2, and 3, if there exist positive-definite matrices $\mathbf{P}$ and $\mathbf{Q}$ such that the following LMI holds,

$$
\left[\begin{array}{cccc}
\Psi_{(1,1)} & \frac{1}{2} \bar{h}_{2} k_{m} k_{s} & 0 & 0 \\
-\bar{h}_{2} \mathbf{P} & 0 & 0 \\
* & \Psi_{(3,3)} & \frac{1}{2} \bar{h}_{1} k_{m} k_{s} \\
* & * & -\bar{h}_{1} \mathbf{Q}
\end{array}\right]<0
$$

where

$$
\begin{aligned}
& \Psi_{(1,1)}=-\alpha_{m} k_{s} k_{n} \mathbf{I}+\bar{h}_{1} \mathbf{Q} \\
& \Psi_{(3,3)}=-\left(\alpha_{s}+m\right) \frac{k_{m}}{k_{n}} \mathbf{I}+\bar{h}_{2} \mathbf{P}
\end{aligned}
$$

and $*$ represents the transpose of corresponding matrix, then $\phi \in \mathscr{L}_{\infty}$.

Proof. First, a positive-definite functional $V=V_{1}+V_{2}+V_{3}+$ $V_{4}+V_{5}>0$ is proposed in order to analyze its evolution along the system trajectories. The first four subfunctionals depend on $\phi$ and are defined in the following manner:

$$
\begin{aligned}
& V_{1}=\frac{1}{2} k_{s} k_{n} \dot{\mathbf{x}}^{\mathrm{T}}\left(\mathbf{M}+m_{h} \mathbf{I}\right) \dot{\mathbf{x}} \\
& V_{2}=\frac{1}{2} \frac{k_{m} k_{s}}{k_{n}}\left(k_{n} \mathbf{x}-\boldsymbol{\eta}\right)^{\mathrm{T}}\left(k_{n} \mathbf{x}-\boldsymbol{\eta}\right) \\
& V_{3}=\alpha_{e} \frac{1}{2} \frac{k_{m}}{k_{n}} \boldsymbol{\eta}^{\mathrm{T}} \boldsymbol{\eta} \\
& V_{4}=\left(m+m_{e}\right) \gamma \frac{1}{2} \frac{k_{m}}{k_{n}} \mathbf{z}^{\mathrm{T}} \mathbf{z} .
\end{aligned}
$$

The time derivative of $V_{1}$ along the master dynamics (2), taking into account Properties 1 and 2 and considering (9), is the following one:

$$
\begin{aligned}
\dot{V}_{1}= & \frac{1}{2} k_{s} k_{n} \dot{\mathbf{x}}^{\mathrm{T}} \dot{\mathbf{M}} \dot{\mathbf{x}}+k_{s} k_{n} \dot{\mathbf{x}}^{\mathrm{T}}\left(\mathbf{M}+m_{h} \mathbf{I}\right) \ddot{\mathbf{x}} \\
= & \frac{1}{2} k_{s} k_{n} \dot{\mathbf{x}}^{\mathrm{T}}(\dot{\mathbf{M}}-2 \mathbf{C}) \dot{\mathbf{x}}+k_{s} k_{n} \dot{\mathbf{x}}^{\mathrm{T}} \mathbf{f}_{\mathbf{m}} \\
& +k_{s} k_{n} \dot{\mathbf{x}}^{\mathrm{T}}\left(-\alpha_{h} \dot{\mathbf{x}}+\mathbf{f}_{\mathbf{h}}^{*}\right) \\
= & k_{s} k_{n} \dot{\mathbf{x}}^{\mathrm{T}} \mathbf{f}_{\mathbf{m}}+k_{s} k_{n} \dot{\mathbf{x}}^{\mathrm{T}}\left(-\alpha_{h} \dot{\mathbf{x}}+\mathbf{f}_{\mathbf{h}}^{*}\right) .
\end{aligned}
$$
yields

Now, if the control action $\mathbf{f}_{\mathbf{m}}$ (15) is included in (22), it

$$
\begin{aligned}
\dot{V}_{1}= & k_{s} k_{n} \dot{\mathbf{x}}^{\mathrm{T}}\left(-k_{m}\left(\mathbf{x}-k_{n}^{-1} \boldsymbol{\eta}\left(t-h_{2}\right)\right)-\alpha_{m} \dot{\mathbf{x}}\right) \\
& +k_{s} k_{n} \dot{\mathbf{x}}^{\mathrm{T}}\left(-\alpha_{h} \dot{\mathbf{x}}+\mathbf{f}_{\mathbf{h}}^{*}\right) \\
= & -k_{m} k_{s} \dot{\mathbf{x}}^{\mathrm{T}}\left(k_{n} \mathbf{x}-\boldsymbol{\eta}\left(t-h_{2}\right)+\boldsymbol{\eta}-\boldsymbol{\eta}\right) \\
& -\left(\alpha_{m}+\alpha_{h}\right) k_{s} k_{n} \dot{\mathbf{x}}^{\mathrm{T}} \dot{\mathbf{x}}+k_{s} k_{n} \dot{\mathbf{x}}^{\mathrm{T}} \mathbf{f}_{\mathbf{h}}^{*} \\
= & -k_{m} k_{s} \dot{\mathbf{x}}^{\mathrm{T}}\left(k_{n} \mathbf{x}-\boldsymbol{\eta}\right)-k_{m} k_{s} \dot{\mathbf{x}}^{\mathrm{T}} \int_{t-h_{2}}^{t} \dot{\boldsymbol{\eta}}(\xi) \mathrm{d} \xi \\
& -\left(\alpha_{m}+\alpha_{h}\right) k_{s} k_{n} \dot{\mathbf{x}}^{\mathrm{T}} \dot{\mathbf{x}}+k_{s} k_{n} \dot{\mathbf{x}}^{\mathrm{T}} \mathbf{f}_{\mathbf{h}}^{*} .
\end{aligned}
$$


Next, $\dot{V}_{2}$ is obtained from (19) considering (14):

$$
\begin{aligned}
\dot{V}_{2}= & \frac{k_{m} k_{s}}{k_{n}}\left(k_{n} \mathbf{x}-\boldsymbol{\eta}\right)^{\mathrm{T}}\left(k_{n} \dot{\mathbf{x}}-\dot{\boldsymbol{\eta}}\right) \\
= & k_{m} k_{s}\left(k_{n} \mathbf{x}-\boldsymbol{\eta}\right)^{\mathrm{T}} \dot{\mathbf{x}}-\frac{k_{m} k_{s}}{k_{n}}\left(k_{n} \mathbf{x}-\boldsymbol{\eta}\right)^{\mathrm{T}} \mathbf{z} \\
& -\gamma \frac{k_{m} k_{s}}{k_{n}}\left(k_{n} \mathbf{x}-\boldsymbol{\eta}\right)^{\mathrm{T}} \dot{\mathbf{z}} .
\end{aligned}
$$

On the other hand, $\dot{V}_{3}$ is computed in a similar way from (20), taking into account (14):

$$
\dot{V}_{3}=\alpha_{e} \frac{k_{m}}{k_{n}} \boldsymbol{\eta}^{\mathrm{T}} \dot{\boldsymbol{\eta}}=\alpha_{e} \frac{k_{m}}{k_{n}} \boldsymbol{\eta}^{\mathrm{T}} \mathbf{z}+\gamma \alpha_{e} \frac{k_{m}}{k_{n}} \boldsymbol{\eta}^{\mathrm{T}} \dot{\mathbf{z}} .
$$

Moreover, $\dot{V}_{4}$ can be written including (12) in (7) and considering (10) and (14), in the following way:

$$
\begin{aligned}
\dot{V}_{4}= & \left(m+m_{e}\right) \gamma \frac{k_{m}}{k_{n}} \mathbf{z}^{\mathrm{T}} \dot{\mathbf{z}} \\
= & \left(m+m_{e}\right) \gamma \frac{k_{m}}{k_{n}} \mathbf{z}^{\mathrm{T}}\left(\frac{\dot{\boldsymbol{\eta}}}{\gamma}-\frac{\mathbf{z}}{\gamma}\right) \\
= & \frac{k_{m}}{k_{n}} \mathbf{z}^{\mathrm{T}}\left(\mathbf{f}_{\mathbf{s}}-\alpha_{e} \boldsymbol{\eta}+\mathbf{f}_{\mathbf{e}}^{*}\right)-\left(m+m_{e}\right) \frac{k_{m}}{k_{n}} \mathbf{z}^{\mathrm{T}} \mathbf{z} \\
= & \frac{k_{m}}{k_{n}} \mathbf{z}^{\mathrm{T}}\left(k_{s}\left(k_{n} \mathbf{x}\left(t-h_{1}\right)-\boldsymbol{\eta}\right)-\alpha_{s} \mathbf{z}\right) \\
& +\frac{k_{m}}{k_{n}} \mathbf{z}^{\mathrm{T}}\left(-\alpha_{e} \boldsymbol{\eta}+\mathbf{f}_{\mathbf{e}}^{*}\right)-\left(m+m_{e}\right) \frac{k_{m}}{k_{n}} \mathbf{z}^{\mathrm{T}} \mathbf{z} \\
= & \frac{k_{m} k_{s}}{k_{n}} \mathbf{z}^{\mathrm{T}}\left(k_{n} \mathbf{x}\left(t-h_{1}\right)-\boldsymbol{\eta}+k_{n} \mathbf{x}-k_{n} \mathbf{x}\right) \\
& -\left(\alpha_{s}+m+m_{e}\right) \frac{k_{m}}{k_{n}} \mathbf{z}^{\mathrm{T}} \mathbf{z}-\alpha_{e} \frac{k_{m}}{k_{n}} \mathbf{z}^{\mathrm{T}} \boldsymbol{\eta}+\frac{k_{m}}{k_{n}} \mathbf{z}^{\mathrm{T}} \mathbf{f}_{\mathbf{e}}^{*} \\
= & \frac{k_{m} k_{s}}{k_{n}} \mathbf{z}^{\mathrm{T}}\left(k_{n} \mathbf{x}-\boldsymbol{\eta}\right)-k_{m} k_{s} \mathbf{z}^{\mathrm{T}} \int_{t-h_{1}}^{t} \dot{\mathbf{x}}^{(\xi)} \mathrm{d} \xi \\
& -\left(\alpha_{s}+m+m_{e}\right) \frac{k_{m}}{k_{n}} \mathbf{z}^{\mathrm{T}} \mathbf{z}-\alpha_{e} \frac{k_{m}}{k_{n}} \mathbf{z}^{\mathrm{T}} \boldsymbol{\eta}+\frac{k_{m}}{k_{n}} \mathbf{z}^{\mathrm{T}} \mathbf{f}_{\mathbf{e}}^{*} .
\end{aligned}
$$

It is possible to appreciate in (23) and (26) that there are terms like $-k_{m} k_{s} \mathbf{z}^{\mathrm{T}} \int_{t-h_{1}}^{t} \dot{\mathbf{x}}(\xi) \mathrm{d} \xi$ which make stability analysis difficult; to solve this, $V_{5}$ is proposed as follows:

$$
\begin{aligned}
V_{5}= & \int_{-\bar{h}_{2}}^{0} \int_{t+\theta}^{t} \mathbf{z}^{\mathrm{T}}(\xi) \mathbf{P z}(\xi) \mathrm{d} \xi \mathrm{d} \theta \\
& +\int_{-\bar{h}_{1}}^{0} \int_{t+\theta}^{t} \dot{\mathbf{x}}^{\mathrm{T}}(\xi) \mathbf{Q} \dot{\mathbf{x}}(\xi) \mathrm{d} \xi \mathrm{d} \theta
\end{aligned}
$$

where $\mathbf{P}$ and $\mathbf{Q}$ are positive-definite matrices.
From (27) and considering Assumption 2, $\dot{V}_{5}$ can be computed by

$$
\begin{aligned}
\dot{V}_{5}= & \bar{h}_{2} \mathbf{z}^{\mathrm{T}} \mathbf{P} \mathbf{z}-\int_{t-\bar{h}_{2}}^{t} \mathbf{z}^{\mathrm{T}}(\xi) \mathbf{P z}(\xi) \mathrm{d} \xi+\bar{h}_{1} \dot{\mathbf{x}}^{\mathrm{T}} \mathbf{Q} \dot{\mathbf{x}} \\
& -\int_{t-\bar{h}_{1}}^{t} \dot{\mathbf{x}}^{\mathrm{T}}(\xi) \mathbf{Q} \dot{\mathbf{x}}(\xi) \mathrm{d} \xi, \\
\dot{V}_{5} \leq & \bar{h}_{2} \mathbf{z}^{\mathrm{T}} \mathbf{P} \mathbf{z}-\int_{t-h_{2}}^{t} \mathbf{z}^{\mathrm{T}}(\xi) \mathbf{P z}(\xi) \mathrm{d} \xi+\bar{h}_{1} \dot{\mathbf{x}}^{\mathrm{T}} \mathbf{Q} \dot{\mathbf{x}} \\
& -\int_{t-h_{1}}^{t} \dot{\mathbf{x}}^{\mathrm{T}}(\xi) \mathbf{Q} \dot{\mathbf{x}}(\xi) \mathrm{d} \xi .
\end{aligned}
$$

On the other hand, each term of (23) and (26) that includes a delayed variable can be conveniently joined using Lemma 3 with one term of $\dot{V}_{5}$. The second term of (23) and the second term of (28) can be linked, considering (14):

$$
\begin{aligned}
- & k_{m} k_{s} \dot{\mathbf{x}}^{\mathrm{T}} \int_{t-h_{2}}^{t} \dot{\boldsymbol{\eta}}(\xi) \mathrm{d} \xi-\int_{t-h_{2}}^{t} \mathbf{z}^{\mathrm{T}}(\xi) \mathbf{P} \mathbf{z}(\xi) \mathrm{d} \xi \\
= & -k_{m} k_{s} \dot{\mathbf{x}}^{\mathrm{T}} \int_{t-h_{2}}^{t} \mathbf{z}(\xi) \mathrm{d} \xi-\int_{t-h_{2}}^{t} \mathbf{z}^{\mathrm{T}}(\xi) \mathbf{P} \mathbf{z}(\xi) \mathrm{d} \xi \\
& -\gamma k_{m} k_{s} \dot{\mathbf{x}}^{\mathrm{T}} \int_{t-h_{2}}^{t} \dot{\mathbf{z}}(\xi) \mathrm{d} \xi \\
\leq & \frac{\bar{h}_{2} k_{m}^{2}}{4 k_{s}^{-2}} \dot{\mathbf{x}}^{\mathrm{T}} \mathbf{P}^{-1} \dot{\mathbf{x}}-\gamma k_{m} k_{s} \dot{\mathbf{x}}^{\mathrm{T}} \int_{t-h_{2}}^{t} \dot{\mathbf{z}}(\xi) \mathrm{d} \xi .
\end{aligned}
$$

In the same manner, the second term of (26) and the fourth term of (28) can be combined to establish the following:

$$
\begin{aligned}
& -k_{m} k_{s} \mathbf{z}^{\mathrm{T}} \int_{t-h_{1}}^{t} \dot{\mathbf{x}}(\xi) \mathrm{d} \xi-\int_{t-h_{1}}^{t} \dot{\mathbf{x}}^{\mathrm{T}}(\xi) \mathbf{Q} \dot{\mathbf{x}}(\xi) \mathrm{d} \xi \\
& \quad \leq \frac{\bar{h}_{1} k_{m}^{2}}{4 k_{s}^{-2}} \mathbf{z}^{\mathrm{T}} \mathbf{Q}^{-1} \mathbf{z} .
\end{aligned}
$$

Finally, $\dot{V}$ can be written associating (23)-(26) and (28)(30), disregarding the terms with the infinitesimal $\gamma$ and neglecting $\alpha_{h}$ and $m_{e}$, in the following way:

$$
\begin{aligned}
\dot{V}= & \dot{V}_{1}+\dot{V}_{2}+\dot{V}_{3}+\dot{V}_{4}+\dot{V}_{5} \\
\leq & \dot{\mathbf{x}}^{\mathrm{T}}\left[-\alpha_{m} k_{s} k_{n} \mathbf{I}+\bar{h}_{1} \mathbf{Q}+\frac{\bar{h}_{2} k_{m}^{2}}{4 k_{s}^{-2}} \mathbf{P}^{-1}\right] \dot{\mathbf{x}} \\
& +\mathbf{z}^{\mathrm{T}}\left[-\left(\alpha_{s}+m\right) \frac{k_{m}}{k_{n}} \mathbf{I}+\bar{h}_{2} \mathbf{P}+\frac{\bar{h}_{1} k_{m}^{2}}{4 k_{s}^{-2}} \mathbf{Q}^{-1}\right] \mathbf{z} \\
& +k_{s} k_{n} \dot{\mathbf{x}}^{\mathrm{T}} \mathbf{f}_{\mathbf{h}}^{*}+k_{m} k_{n}^{-1} \mathbf{z}^{\mathrm{T}} \mathbf{f}_{\mathbf{e}}^{*},
\end{aligned}
$$

where $\mathbf{I} \in \mathfrak{R}^{3 \times 3}$ is the identity matrix. 


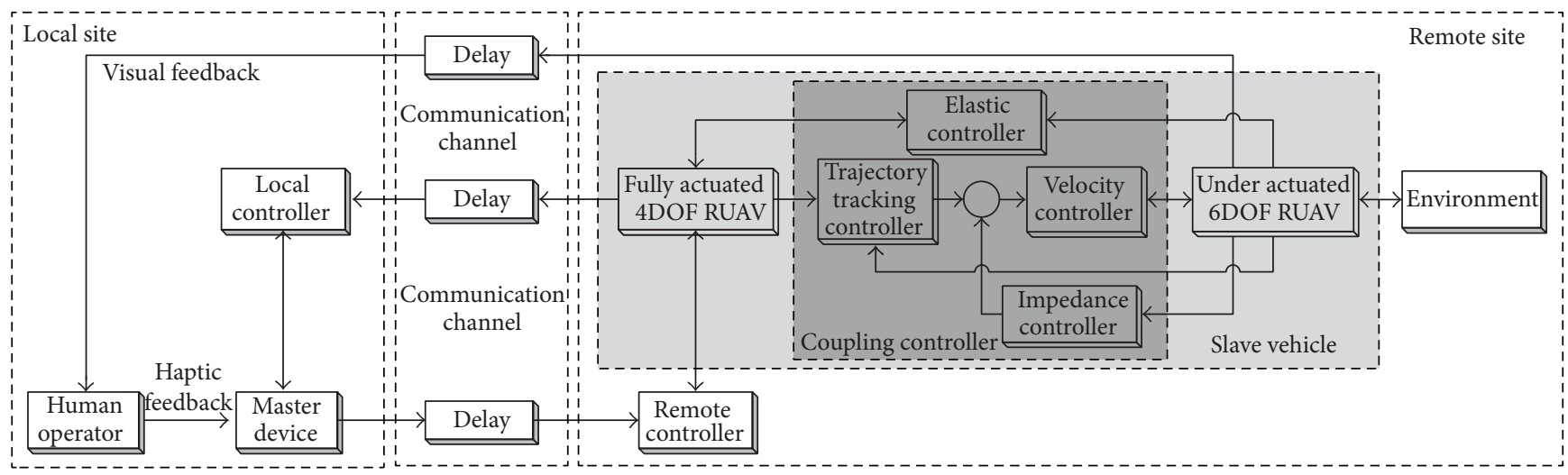

FIgURE 4: Complete bilateral teleoperation system. The slave vehicle block is composed of the fully actuated 4DOF RUAV and the underactuated 6DOF RUAV (minihelicopter) both attached to a coupling controller.

LMI conditions (16) are equivalent to the following two inequalities:

$$
\begin{array}{r}
-\alpha_{m} k_{s} k_{n} \mathbf{I}+\bar{h}_{1} \mathbf{Q}+\frac{\bar{h}_{2} k_{m}^{2}}{4 k_{s}^{-2}} \mathbf{P}^{-1}<0 \\
-\left(\alpha_{s}+m\right) \frac{k_{m}}{k_{n}} \mathbf{I}+\bar{h}_{2} \mathbf{P}+\frac{\bar{h}_{1} k_{m}^{2}}{4 k_{s}^{-2}} \mathbf{Q}^{-1}<0 .
\end{array}
$$

Given $\bar{h}_{1}$ and $\bar{h}_{2}$ maximum delays and slave vehicle mass $m$, the controllers parameters $\left(k_{m}, k_{s}, \alpha_{m}, \alpha_{s}\right.$, and $\left.k_{n}\right)$ can be selected according to LMI conditions (16) to ensure that the two inequalities (32) are satisfied; that is, the first two terms of (31) are negative definite and therefore the variables $\dot{\mathbf{x}},\left(k_{n} \mathbf{x}-\right.$ $\boldsymbol{\eta}), \boldsymbol{\eta}$, and $\mathbf{z}$ are bounded $\left(\phi \in \mathscr{L}_{\infty}\right)$. The proof is completed.

Remark 5. If the exogenous force of the human operator $\mathbf{f}_{\mathbf{h}}^{*}$ and environment $\mathbf{f}_{\mathbf{e}}^{*}$ are null $\left(\bar{f}_{h}^{*}=\bar{f}_{e}^{*}=0\right)$, then the system is stable. For this particular case, Barbalat's lemma can be used in (31), taking into account Assumptions 1 and 2, Property 3, and the fact that $\phi \in \mathscr{L}_{\infty}$ (and $\mathbf{x} \in \mathscr{L}_{\infty}$ ); it is possible to deduce that $\ddot{\mathbf{x}}$ and $\dot{\mathbf{z}}$ are bounded; then, $\ddot{V}$ is bounded too. Finally, $\dot{\mathbf{x}}$ and $\mathbf{z}$ will tend to zero as $t \rightarrow \infty$.

\section{Complete Bilateral Teleoperation System}

To test the performance of the teleoperation system a HITL simulation will be executed; there, a human operator will drive a very realistic $6 \mathrm{DOF}$ model of a minihelicopter (extracted from [24]) through an obstructed environment. The helicopter model considers nonideal dynamics, for example, flapping, drag, and actuators' dynamic, and precisely describes the vehicle's motion for both hovering and low-speed translational flight.

The minihelicopter needs to be outside the closed-loop bilateral teleoperation system for the stability analysis of previous section to remain valid; hence, a coupling between the $4 \mathrm{DOF}$ and $6 \mathrm{DOF}$ RUAVs is proposed. The complete bilateral teleoperation system can be observed in Figure 4.
The coupling controller has two main functions: allow the 6DOF RUAV to track the trajectory of the 4DOF RUAV and transmit the interactions between the minihelicopter and its environment to the 4DOF RUAV. In addition, obstacle avoidance is incorporated to the minihelicopter. Next, each part of the coupling controller is explained.

7.1. Trajectory Tracking Controller. Inspired by [30], the $4 \mathrm{DOF}$ RUAV is treated as a moving reference frame $\langle r\rangle$ (axes $\left.o_{E} x_{E} y_{E} z_{E}\right)$ whose trajectory is the reference for the helicopter frame $\langle h\rangle$ (axes $o_{E} x_{E} y_{E} z_{E}$ ) attached to the 6DOF as seen in Figure 5. The axes $\left(o_{E} x_{E} y_{E} z_{E}\right)$ of the frame $\langle h\rangle$ are not the actual body axes of the minihelicopter but 4DOF RUAV-like axes. The kinematics of the helicopter frame $\langle h\rangle$ is equal to the kinematics of the reference frame $\langle r\rangle$ :

$$
\left[\begin{array}{c}
\dot{x}_{i} \\
\dot{y}_{i} \\
\dot{z}_{i} \\
\dot{\psi}_{i}
\end{array}\right]=\left[\begin{array}{cccc}
\cos \psi_{i} & -\sin \psi_{i} & 0 & 0 \\
\sin \psi_{i} & \cos \psi_{i} & 0 & 0 \\
0 & 0 & 1 & 0 \\
0 & 0 & 0 & 1
\end{array}\right]\left[\begin{array}{c}
v_{x_{E}}^{i} \\
v_{y_{E}}^{i} \\
v_{z_{E}}^{i} \\
\omega_{z_{E}}^{i}
\end{array}\right]
$$

being $i=r$ for the reference frame and $i=h$ for the helicopter frame.

Considering the RUAVs' kinematics model (33), the trajectory tracking problem corresponds to the design of a control law that allows the helicopter frame to reach and automatically follow a path described by the reference frame according to the 4DOF RUAV velocities. The controller must generate the flight commands $v_{x_{E}}^{h}, v_{y_{E}}^{h}, v_{z_{E}}^{h}$, and $\omega_{z_{E}}^{h}$ such that

$$
\left(X_{r}(t)-X_{h}(t)\right) \longrightarrow 0,
$$

where $X_{h}=\left[\begin{array}{llll}x_{h} & y_{h} & z_{h} & \psi_{h}\end{array}\right]^{\mathrm{T}}$ and $X_{r}=\left[\begin{array}{llll}x_{r} & y_{r} & z_{r} & \psi_{r}\end{array}\right]^{\mathrm{T}}$.

First, the following errors are defined:

$$
\mathbf{e}=\left[\begin{array}{l}
e_{1} \\
e_{2} \\
e_{3} \\
e_{4}
\end{array}\right]=\left[\begin{array}{cccc}
\cos \psi_{h} & \sin \psi_{h} & 0 & 0 \\
-\sin \psi_{h} & \cos \psi_{h} & 0 & 0 \\
0 & 0 & 1 & 0 \\
0 & 0 & 0 & 1
\end{array}\right]\left[\begin{array}{c}
x_{r}-x_{h} \\
y_{r}-y_{h} \\
z_{r}-z_{h} \\
\psi_{r}-\psi_{h}
\end{array}\right] .
$$




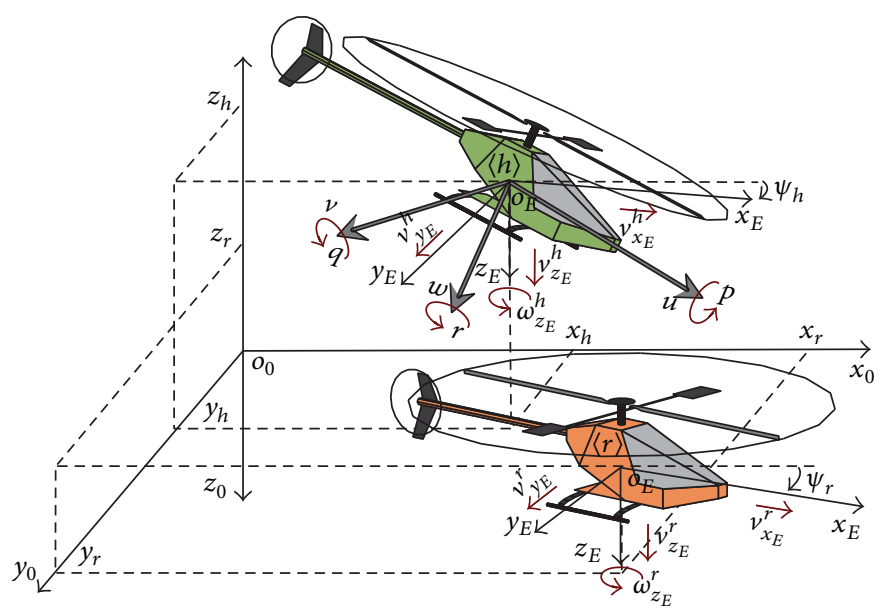

Figure 5: Position and orientation of the 4DOF and 6DOF RUAVs along with helicopter $\langle h\rangle$ and reference $\langle r\rangle$ frames.

The states $e_{i}$ are errors $\left(x_{r}-x_{h}\right),\left(y_{r}-y_{h}\right),\left(z_{r}-z_{h}\right)$, and $\left(\psi_{r}-\psi_{h}\right)$ expressed in the helicopter frame $\langle h\rangle$. The time derivative of $\mathbf{e}$ is the following:

$$
\dot{\mathbf{e}}=\left[\begin{array}{cccc}
0 & \omega_{z_{E}}^{h} & 0 & 0 \\
-\omega_{z_{E}}^{h} & 0 & 0 & 0 \\
0 & 0 & 0 & 0 \\
0 & 0 & 0 & 0
\end{array}\right]\left[\begin{array}{l}
e_{1} \\
e_{2} \\
e_{3} \\
e_{4}
\end{array}\right]+\left[\begin{array}{llll}
1 & 0 & 0 & 0 \\
0 & 1 & 0 & 0 \\
0 & 0 & 1 & 0 \\
0 & 0 & 0 & 1
\end{array}\right]\left[\begin{array}{l}
\mu_{1} \\
\mu_{2} \\
\mu_{3} \\
\mu_{4}
\end{array}\right]
$$

being

$$
\begin{aligned}
& \mu_{1}=-v_{x_{E}}^{h}+v_{x_{E}}^{r} \cos e_{4}-v_{y_{E}}^{r} \sin e_{4} \\
& \mu_{2}=-v_{y_{E}}^{h}+v_{x_{E}}^{r} \sin e_{4}+v_{y_{E}}^{r} \cos e_{4} \\
& \mu_{3}=-v_{z_{E}}^{h}+v_{z_{E}}^{r} \\
& \mu_{4}=-\omega_{z_{E}}^{h}+\omega_{z_{E}}^{r} .
\end{aligned}
$$

Now, the following Lyapunov candidate is considered:

$$
V(\mathbf{e})=\frac{1}{2}\left(e_{1}^{2}+e_{2}^{2}+e_{3}^{2}+e_{4}^{2}\right)
$$

The time derivative $\dot{V}(\mathbf{e})$ along the system states (35) is given by

$$
\begin{aligned}
\dot{V}(\mathbf{e})= & e_{1} \dot{e}_{1}+e_{2} \dot{e}_{2}+e_{3} \dot{e}_{3}+e_{4} \dot{e}_{4} \\
\dot{V}(\mathbf{e})= & e_{1}\left(\mu_{1}+\omega_{n} e_{2}\right)+e_{2}\left(\mu_{2}-\omega_{n} e_{1}\right)+e_{3}\left(\mu_{3}\right) \\
& +e_{4}\left(\mu_{4}\right) \\
\dot{V}(\mathbf{e})= & e_{1}\left(\mu_{1}\right)+e_{2}\left(\mu_{2}\right)+e_{3}\left(\mu_{3}\right)+e_{4}\left(\mu_{4}\right) .
\end{aligned}
$$

If the flight commands in (37) are selected as follows

$$
\begin{aligned}
& v_{x_{E}}^{h}=k_{1} \tanh \left(k_{5} e_{1}\right)+v_{x_{E}}^{r} \cos e_{4}-v_{y_{E}}^{r} \sin e_{4} \\
& v_{y_{E}}^{h}=k_{2} \tanh \left(k_{5} e_{2}\right)+v_{x_{E}}^{r} \sin e_{4}+v_{y_{E}}^{r} \cos e_{4} \\
& v_{z_{E}}^{h}=k_{3} \tanh \left(k_{5} e_{3}\right)+v_{z_{E}}^{r} \\
& \omega_{z_{E}}^{h}=k_{4} e_{4}+\omega_{z_{E}}^{r}
\end{aligned}
$$

with $k_{i}>0$, and then (37) is replaced in (39), the final expression for $\dot{V}(\mathbf{e})$ is

$$
\begin{aligned}
\dot{V}(\mathbf{e})= & -k_{1} e_{1} \tanh \left(k_{5} e_{1}\right)-k_{2} e_{2} \tanh \left(k_{5} e_{2}\right) \\
& -k_{3} e_{3} \tanh \left(k_{5} e_{3}\right)-k_{4} e_{4}^{2},
\end{aligned}
$$

which is a negative definite function. This means that system errors $e_{1}, e_{2}, e_{3}, e_{4}$ asymptotically converge to zero.

7.2. Impedance Controller. To add obstacle avoidance in the coupling controller the following correction to the flight commands (40) is made:

$$
\begin{aligned}
& v_{x_{E} c}^{h}=v_{x_{E}}^{h}-k_{v x} F_{\text {lon }} \\
& v_{y_{E} c}^{h}=v_{y_{E}}^{h}-k_{v y} F_{\text {lat }} \\
& v_{z_{E} c}^{h}=v_{z_{E}}^{h}-k_{v z} F_{\text {ver }} \\
& \omega_{z_{E} c}^{h}=\omega_{z_{E}}^{h} .
\end{aligned}
$$




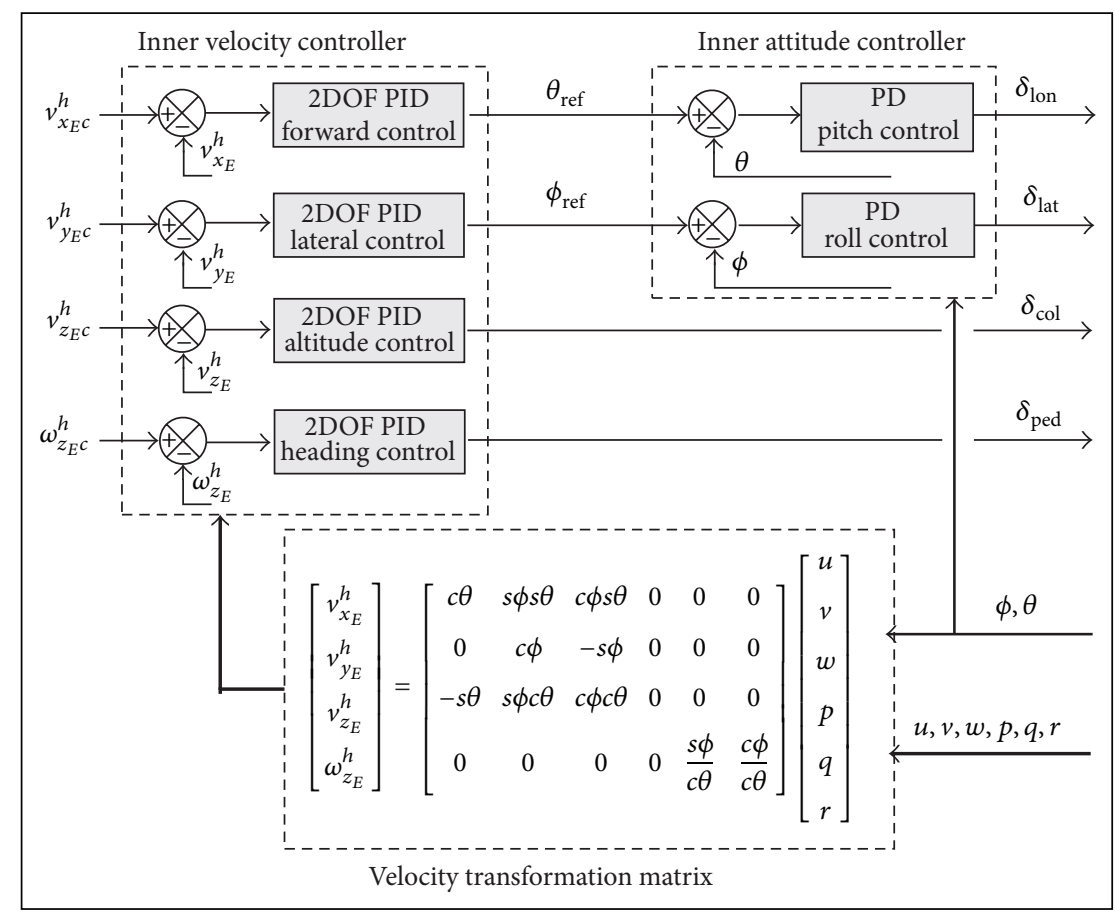

FIGURE 6: Block diagram of the velocity controller.

The fictitious force is calculated using a simulated $3 \mathrm{D}$ laser sensor which measures distance between the minihelicopter and the obstacles. The force is obtained as follows:

$$
\left[\begin{array}{c}
F_{\text {lon }} \\
F_{\text {lat }} \\
F_{\text {ver }}
\end{array}\right]=\left[\begin{array}{c}
k_{\text {lon }} \sum_{i=1}^{j} f_{i} \cos \phi_{i} \cos \varphi_{i} \\
k_{\text {lat }} \sum_{i=1}^{j} f_{i} \sin \phi_{i} \cos \varphi_{i} \\
k_{\mathrm{ver}} \sum_{i=1}^{j} f_{i} \sin \varphi_{i}
\end{array}\right],
$$

$F_{\text {lon }}, F_{\text {lat }}$, and $F_{\text {ver }}$ being the longitudinal, lateral, and vertical forces, respectively; $k_{\text {lon }}, k_{\text {lat }}$, and $k_{\text {ver }}$ normalize forces to $[0,1]$ for $F_{\text {lon }}$ and $[-1,1]$ for $F_{\text {lat }}$ and $F_{\text {ver }} \cdot f_{i}=a-b d_{i}(t)$ indicates the magnitude of the force for each laser beam $(1 \leq i \leq j)$, with $a, b>0$ such that $a-b d_{\max }=0$ and $a-b d_{\min }=1\left(d_{\min } \leq d_{i} \leq d_{\max }\right)$. Angles $\phi_{i}$ and $\varphi_{i}$ express the direction of each laser beam in the horizontal and vertical plane, respectively.

7.3. Velocity Controller. To let the minihelicopter track the flight commands (42), the velocity controller depicted in Figure 6 is incorporated. It has a velocity transformation matrix that maps the 6DOF actual body axes kinematics $(u, v, w, p, q, r$ in Figure 5) to the helicopter frame $\langle h\rangle$ and a cascade PID architecture to generate the servo signal inputs (see details in [31]).

7.4. Elastic Controller. The interaction between the helicopter and its environment is reflected to the human operator, improving the telepresence, thanks to the elastic controller; moreover, the position and orientation errors between RUAVs are minimized. To achieve both goals, the exogenous environment force $\mathbf{f}_{\mathbf{e}}^{*}$ and an exogenous environment torque $\tau_{e}^{*}$ acting on the 4DOF RUAV are considered to be the following:

$$
\begin{aligned}
\mathbf{f}_{\mathrm{e}}^{*} & =-k_{\mathrm{ep}} \mathbf{E}_{p}, \\
\tau_{e}^{*} & =-k_{\mathrm{eo}} e_{o}
\end{aligned}
$$

$\mathbf{E}_{p}=\left[\begin{array}{lll}e_{1} & e_{2} & e_{3}\end{array}\right]^{\mathrm{T}}$ and $e_{o}=e_{4}$ being the position and orientation errors previously defined in (35). Equation (44) must satisfy $\left|-k_{\mathrm{ep}} \mathbf{E}_{p}\right| \leq \bar{f}_{e}^{*}$ to fulfill the stability analysis of Section 6.

\section{HITL Simulation}

The simulation environment has been developed in Matlab ${ }^{\odot}$, where the human operator's view simulates an onboard camera. The aim of the simulation was to reach eight successive target points (see Figure 7). The calculations of the whole teleoperation system (including RUAVs dynamics) and its connection with the master devices and Matlab ${ }^{\odot}$ are accomplished by a program made in $\mathrm{C}++$.

The teleoperation system's parameters are the following: $\alpha_{m_{\mathrm{C} 1, \mathrm{C} 2 \mathrm{C}, \mathrm{C} 4}}=0,5,20,70\left[\mathrm{Ns} / \mathrm{m}\right.$ ) (obtained using Matlab ${ }^{\odot} \mathrm{LMI}$ toolbox for each of the four different cases of time delay as seen in Table 1), $\alpha_{s}=0.65\left[\mathrm{Ns}^{2} / \mathrm{m}\right], \alpha_{\mathrm{sr}}=0.1\left[\mathrm{Nms}^{2}\right], k_{m}=$ $100[\mathrm{~N} / \mathrm{m}], k_{s}=10[\mathrm{Ns} / \mathrm{m}], k_{\mathrm{sr}}=0.7[\mathrm{Nms}], k_{n_{x, y, z}}=$ $200,100,40[1 / \mathrm{s}], k_{\mathrm{nr}}=0.75[1 / \mathrm{s}], k_{1,2,3,4,5}=10,5,2,1 / \pi, 0.1$, $k_{v x, y, z}=10,3.75,1.5, k_{\mathrm{ep}_{x, y, z}}=20,10,4$, and $k_{\mathrm{eo}}=0.5$. The 4DOF RUAV has mass $m=7.4[\mathrm{~kg}]$ and rotational inertia 

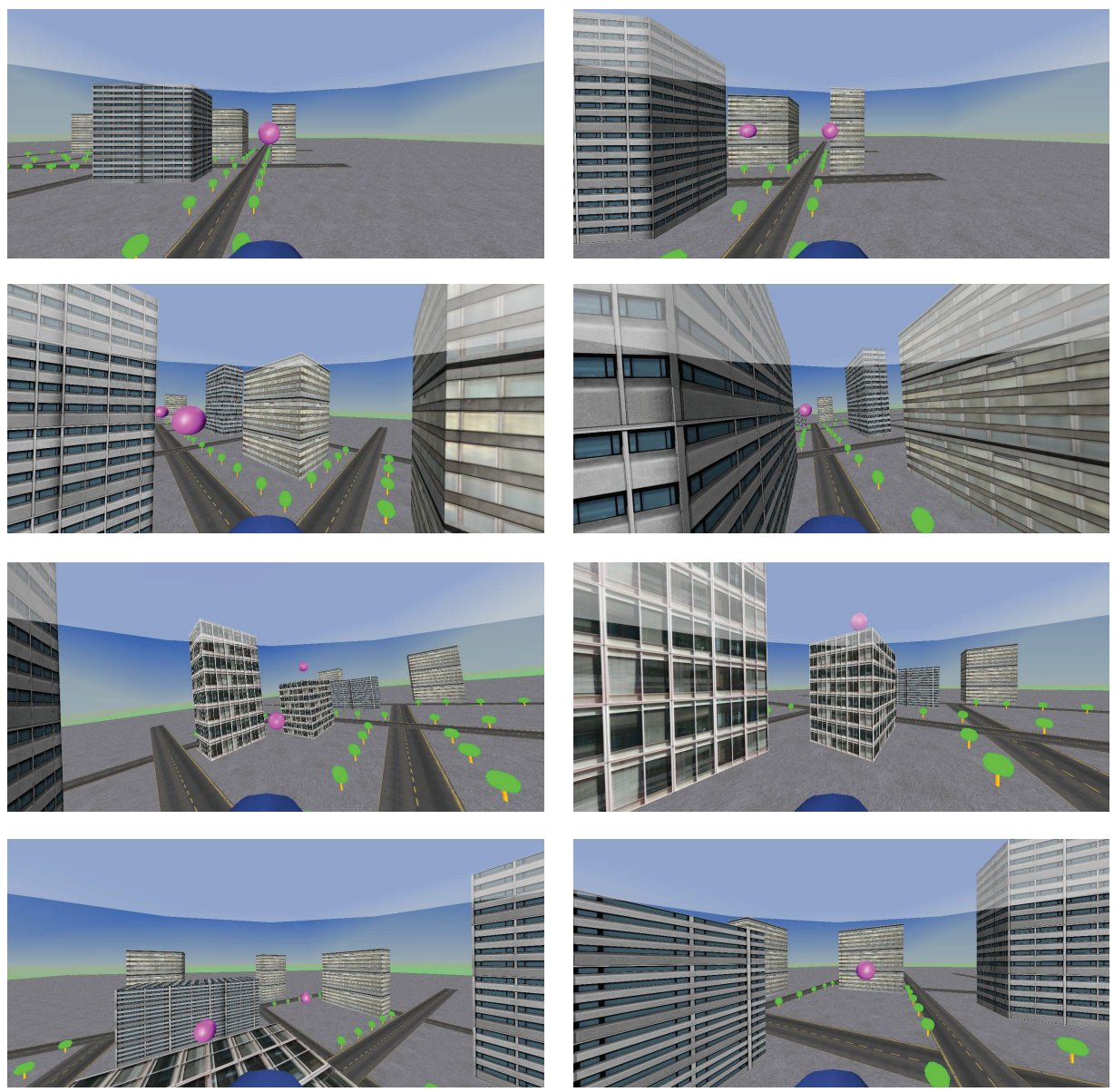

FIGURE 7: Human operator visual feedback of the different target points in the remote environment.

TABLE 1: Average values for time to complete the task (TCT) and mean square translational synchronization error (MSE) for different time delays.

\begin{tabular}{|c|c|c|c|c|c|}
\hline Case & Time delays [s] & TCT [s] & $\operatorname{MSE}_{x}[\mathrm{~m} / \mathrm{s}]$ & $\mathrm{MSE}_{y}[\mathrm{~m} / \mathrm{s}]$ & $\operatorname{MSE}_{z}[\mathrm{~m} / \mathrm{s}]$ \\
\hline 1 & $h_{1}=h_{2}=0$ & 77.86 & 3.93 & 1.4 & 0.13 \\
\hline 2 & $\begin{array}{l}h_{1}=0.1+0.1 \sin (1.26 t) \\
h_{2}=0.1+0.1 \sin (0.63 t)\end{array}$ & 80.69 & 6.86 & 1.98 & 0.15 \\
\hline 3 & $\begin{array}{l}h_{1}=0.2+0.1 \sin (0.63 t) \\
h_{2}=0.3+0.2 \sin (1.26 t)\end{array}$ & 100.14 & 7.41 & 2.43 & 0.14 \\
\hline 4 & $\begin{array}{l}h_{1}=0.5+0.4 \sin (1.26 t) \\
h_{2}=0.4+0.2 \sin (0.63 t)\end{array}$ & 129.93 & 6.87 & 2.4 & 0.16 \\
\hline
\end{tabular}

$i_{r}=0.28\left[\mathrm{kgm}^{2}\right]$, and the 6DOF RUAV has the minihelicopter's nominal parameters (which refer to MIT's X-Cell $.60)$.

8.1. Simulation Results. In Table 1 the time to complete the task (TCT) and mean square translational synchronization error (MSE) for four different time delays are presented. The values correspond to the average of five simulations for each case of time delay. When time delay increases, the TCT gets bigger, as expected. The behaviour of the MSE is much more complicated: when time delays are zero (case 1), all the MSE is due to the dynamics of the RUAV models and the coupling controller, specially the impedance controller because the obstacle avoidance directly modifies the flight commands; when time delay increases (cases 2, 3, and 4), the MSE gets bigger as the synchronism gets worse; however, when the local controller damping $\alpha_{m}$ rises considerably because of time delay $\left(\alpha_{m}=70\right.$ for case 4 so that condition (16) is satisfied), the movement of the master device is slowed down causing the MSE to decrease. The magnitude difference between $\mathrm{MSE}_{x}, \mathrm{MSE}_{y}$, and $\mathrm{MSE}_{z}$ is promoted by the different 


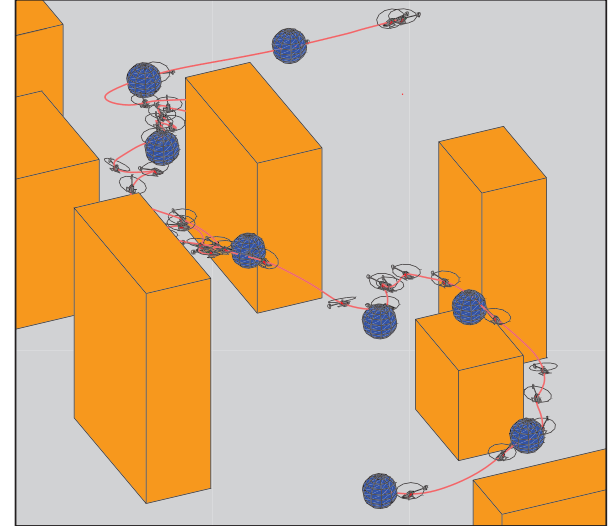

(a)

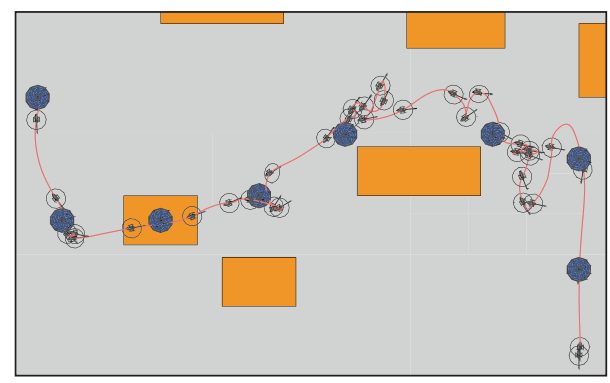

(b)

Figure 8: 3D (a) and 2D (b) views of the 6DOF RUAV trajectory for a simulation with case 4 time delays.

velocity mappings $k_{n_{i}}$, and the invariance in $\mathrm{MSE}_{z}$ appears as a result of better low level PID control (improved immunity to desynchronization) and the fact that there is only one obstacle that seriously affects the vertical axis (see Figure 8).

Figures 8 and 9 present some results obtained in a case 4 HITL simulation $\left(\bar{h}_{1}=0.9[\mathrm{~s}]\right.$ and $\left.\bar{h}_{2}=0.6[\mathrm{~s}]\right)$. Figure 8 shows 3D and 2D views of the trajectory made by the minihelicopter in the simulation task, every 5 seconds the 6DOF RUAV is drawn. In Figure 9, it can be observed that all the teleoperation signals are bounded, and when the environment exogenous force decreases, the synchronization error and force feedback diminish too. It is necessary to highlight the complexity of the performed task, mainly due to the big asymmetric time-varying delay but also by the number of obstacles and the speed of the minihelicopter in the simulation.

\section{Conclusions}

In this paper, a bilateral teleoperation scheme for a RUAV has been proposed. The complete teleoperation system incorporates $\mathrm{P}+\mathrm{d}$ controllers, a trajectory tracking control, an impedance control with obstacle avoidance, an elastic coupling controller, and a cascade PID control architecture. The stability of the system has been analyzed and delay-dependent stability criteria have been obtained under specific LMI conditions. The teleoperation scheme has performed well in the presence of different time-varying delays as shown in the
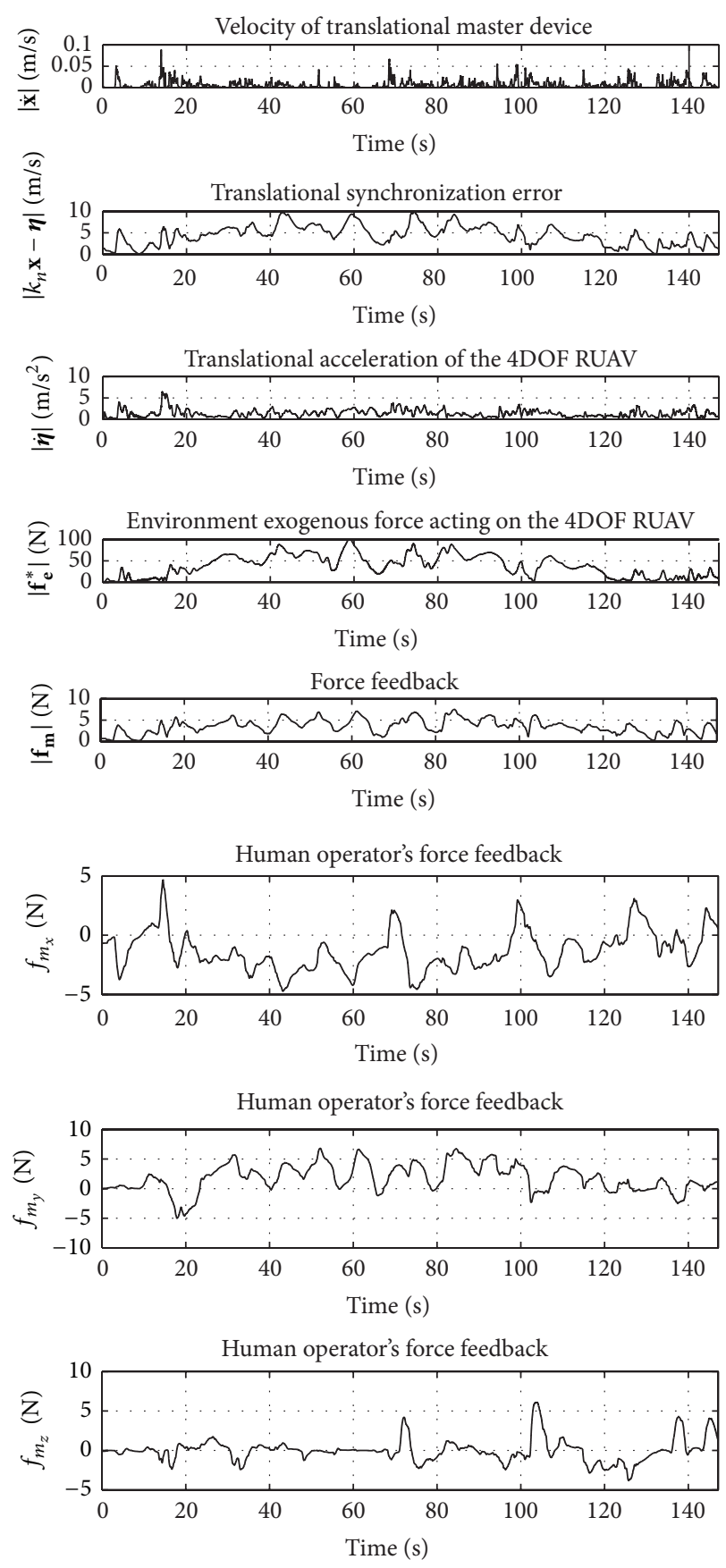

FIgURE 9: Teleoperation system signals, environment exogenous force, and human operator haptic feedback for a simulation with case 4 time delays.

HITL simulation results. The inclusion of simple controllers in the teleoperation system makes their parameters easy to set, allowing accurately calibrating how the human operator perceives the environment and dynamics of the RUAV through force feedback.

\section{Conflict of Interests}

The authors declare that there is no conflict of interests regarding the publication of this paper. 


\section{Acknowledgments}

The authors would like to thank the AC3E, Universidad Técnica Federico Santa María, Grant FB0008, CONICYT, and the INAUT, Universidad Nacional de San Juan, CONICET, for their support.

\section{References}

[1] B. Ahmed, H. R. Pota, and M. Garratt, "Flight control of a rotary wing UAV using backstepping," International Journal of Robust and Nonlinear Control, vol. 20, no. 6, pp. 639-658, 2010.

[2] F. Kendoul, "Survey of advances in guidance, navigation, and control of unmanned rotorcraft systems," Journal of Field Robotics, vol. 29, no. 2, pp. 315-378, 2012.

[3] S. Stramigioli, R. Mahony, and P. Corke, "A novel approach to haptic tele-operation of aerial robot vehicles," in Proceedings of the IEEE International Conference on Robotics and Automation (ICRA '10), pp. 5302-5308, May 2010.

[4] T. M. Lam, M. Mulder, and M. M. Van Paassen, "Haptic feedback in uninhabited aerial vehicle teleoperation with time delay," Journal of Guidance, Control, and Dynamics, vol. 31, no. 6, pp. 1728-1739, 2008.

[5] S. de Vries, "UAVs and control delays," Tech. Rep. TNO-DV32005-A054, TNO Defence Security and Safety, Soesterberg, The Netherlands, 2005.

[6] S. I. Niculescu, Delay Effects on Stability: A Robust Control Approach, Springer, 2001.

[7] S.-I. Niculescu, D. Taoutaou, and R. Lozano, "Bilateral teleoperation with communication delays," International Journal of Robust and Nonlinear Control, vol. 13, no. 9, pp. 873-883, 2003.

[8] R. J. Anderson and M. W. Spong, "Bilateral control of teleoperators with time delay," IEEE Transactions on Automatic Control, vol. 34, no. 5, pp. 494-501, 1989.

[9] P. F. Hokayem and M. W. Spong, "Bilateral teleoperation: an historical survey," Automatica, vol. 42, no. 12, pp. 2035-2057, 2006.

[10] T. M. Lam, M. Mulder, M. M. VanPaassen, J. A. Mulder, and F. C. T. Van DerHelm, "Force-stiffness feedback in uninhabited aerial vehicle teleoperation with time delay," Journal of Guidance, Control, and Dynamics, vol. 32, no. 3, pp. 821-835, 2009.

[11] G. Niemeyer and J.-J. E. Slotine, "Stable adaptive teleoperation," IEEE Journal of Oceanic Engineering, vol. 16, no. 1, pp. 152-162, 1991.

[12] G. Niemeyer and J.-J. E. Slotine, "Telemanipulation with time delays," The International Journal of Robotics Research, vol. 23, no. 9, pp. 873-890, 2004.

[13] E. J. Rodríguez-Seda, J. J. Troy, C. A. Erignac, P. Murray, D. M. Stipanović, and M. W. Spong, "Bilateral teleoperation of multiple mobile agents: coordinated motion and collision avoidance," IEEE Transactions on Control Systems Technology, vol. 18, no. 4, pp. 984-992, 2010.

[14] P. Giordano, A. Franchi, C. Secchi, and H. H. Bülthoff, "Experiments of passivity-based bilateral aerial teleoperation of a group of UAVs with decentralized velocity synchronization," in Proceedings of the IEEE/RSJ International Conference on Intelligent Robots and Systems, San Francisco, Calif, USA, December 2011.

[15] A. Franchi, C. Secchi, H. I. Son, H. H. Bülthoff, and P. R. Giordano, "Bilateral teleoperation of groups of mobile robots with time-varying topology," IEEE Transactions on Robotics, vol. 28, no. 5, pp. 1019-1033, 2012.
[16] D. Lee, A. Franchi, H. I. Son, C. Ha, H. H. Bulthoff, and P. R. Giordano, "Semiautonomous haptic teleoperation control architecture of multiple unmanned aerial vehicles," IEEE/ASME Transactions on Mechatronics, vol. 18, no. 4, pp. 1334-1345, 2013.

[17] H. I. Son, A. Franchi, L. L. Chuang, J. Kim, H. H. Bülthoff, and P. R. Giordano, "Human-centered design and evaluation of haptic cueing for teleoperation of multiple mobile robots," IEEE Transactions on Cybernetics, vol. 43, no. 2, pp. 597-609, 2013.

[18] D. Lee and D. Xu, "Feedback r-passivity of Lagrangian systems for mobile robot teleoperation," in Proceedings of the IEEE International Conference on Robotics and Automation (ICRA '11), pp. 2118-2123, Shanghai, China, May 2011.

[19] D. Lee and K. Huang, "Passive-set-position-modulation framework for interactive robotic systems," IEEE Transactions on Robotics, vol. 26, no. 2, pp. 354-369, 2010.

[20] A. Y. Mersha, S. Stramigioli, and R. Carloni, "On bilateral teleoperation of aerial robots," IEEE Transactions on Robotics, vol. 30, no. 1, pp. 258-274, 2014.

[21] E. Nũo, L. Basãez, R. Ortega, and M. W. Spong, "Position tracking for non-linear teleoperators with variable time delay," The International Journal of Robotics Research, vol. 28, no. 7, pp. 895-910, 2009.

[22] C.-C. Hua and X. P. Liu, "Delay-dependent stability criteria of teleoperation systems with asymmetric time-varying delays," IEEE Transactions on Robotics, vol. 26, no. 5, pp. 925-932, 2010.

[23] E. Slawiñski and V. Mut, "Pd-like controllers for delayed bilateral teleoperation of manipulators robots," International Journal of Robust and Nonlinear Control, vol. 25, no. 12, pp. 18011815, 2015.

[24] V. Gavrilets, Autonomous aerobatic maneuvering of miniature helicopters [Ph.D. thesis], Department of Aeronautics and Astronautics, Massachusetts Institute of Technology, Cambridge, Mass, USA, 2003.

[25] S. Yin, S. X. Ding, X. Xie, and H. Luo, "A review on basic datadriven approaches for industrial process monitoring," IEEE Transactions on Industrial Electronics, vol. 61, no. 11, pp. 64146428, 2014.

[26] M. Shahbazi, S. F. Atashzar, H. A. Talebi, and R. V. Patel, "Novel cooperative teleoperation framework: multi-master/singleslave system," IEEE/ASME Transactions on Mechatronics, 2014.

[27] A. Shahdi and S. Sirouspour, "Adaptive/robust control for timedelay teleoperation," IEEE Transactions on Robotics, vol. 25, no. 1, pp. 196-205, 2009.

[28] W.-H. Zhu and S. E. Salcudean, "Stability guaranteed teleoperation: an adaptive motion/force control approach," IEEE Transactions on Automatic Control, vol. 45, no. 11, pp. 1951-1969, 2000.

[29] N. N. Krasovskii, Stability of Motion, Stanford University Press, Stanford, Calif, USA, 1963.

[30] Y. Kanayama, Y. Kimura, F. Miyazaki, and T. Noguchi, "A stable tracking control method for an autonomous mobile robot," in Proceedings of the IEEE International Conference on Robotics and Automation, pp. 384-389, May 1990.

[31] L. R. Salinas, E. Slawiñski, and V. A. Mut, "Kinematic nonlinear controller for a miniature helicopter via Lyapunov techniques," Asian Journal of Control, vol. 16, no. 3, pp. 856-870, 2014. 


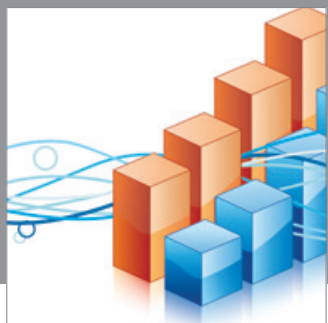

Advances in

Operations Research

mansans

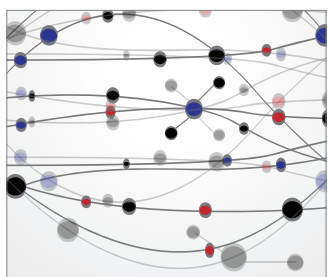

The Scientific World Journal
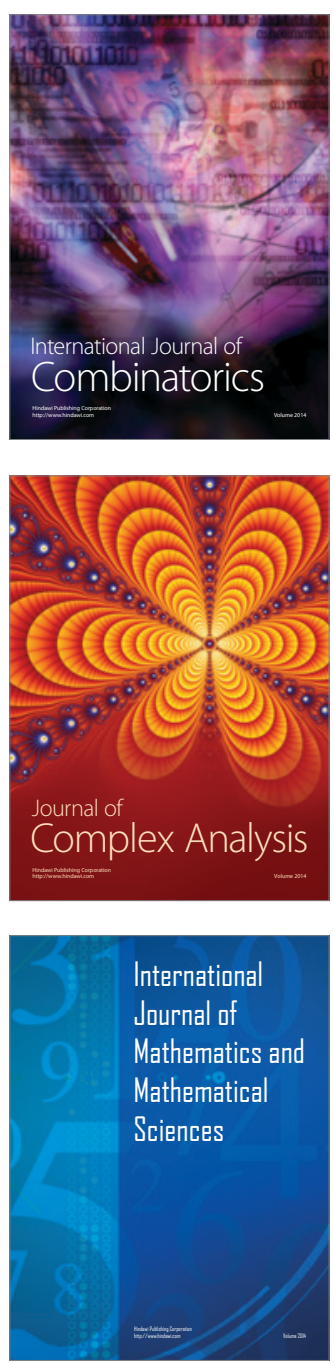
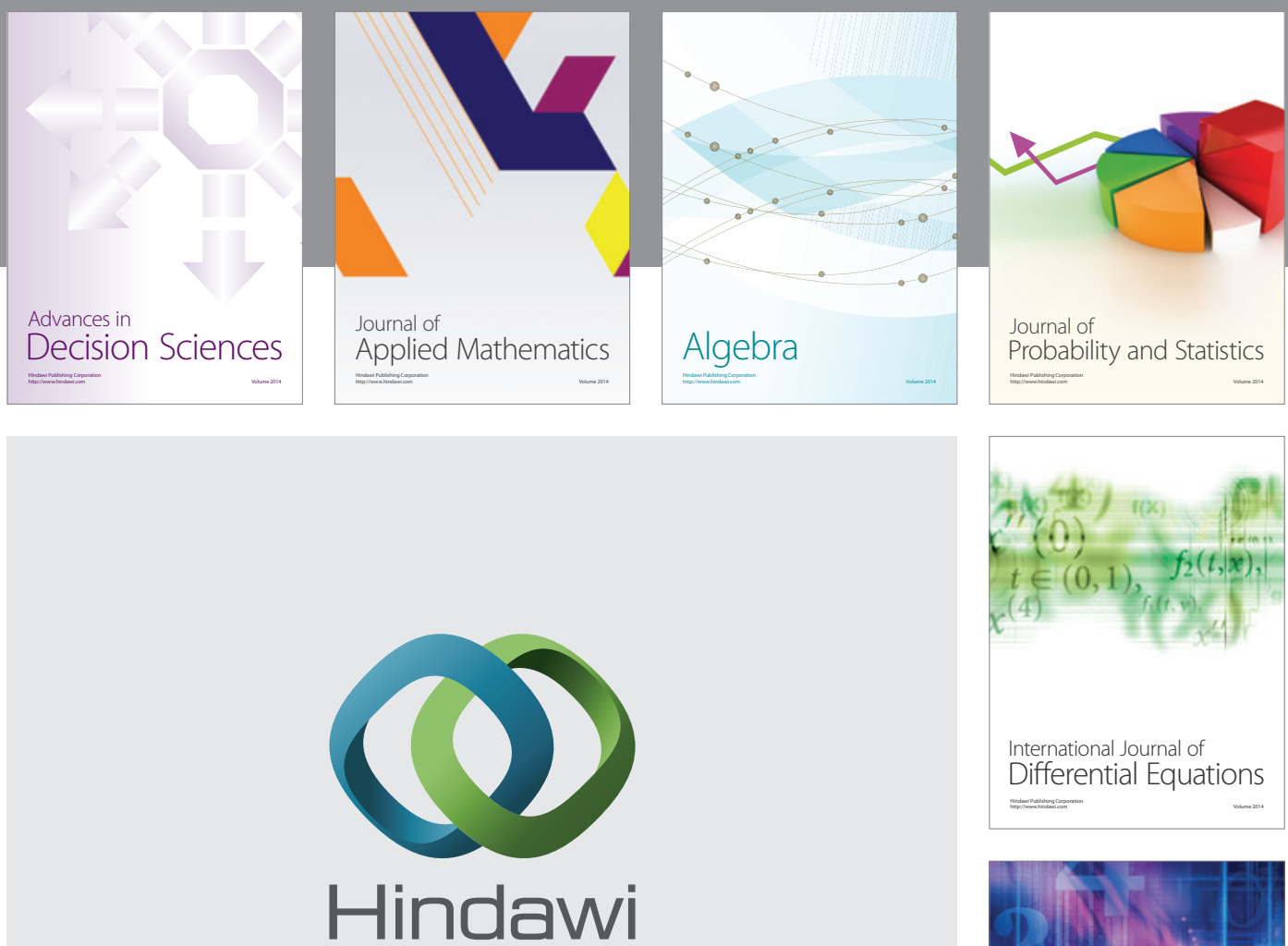

Submit your manuscripts at http://www.hindawi.com
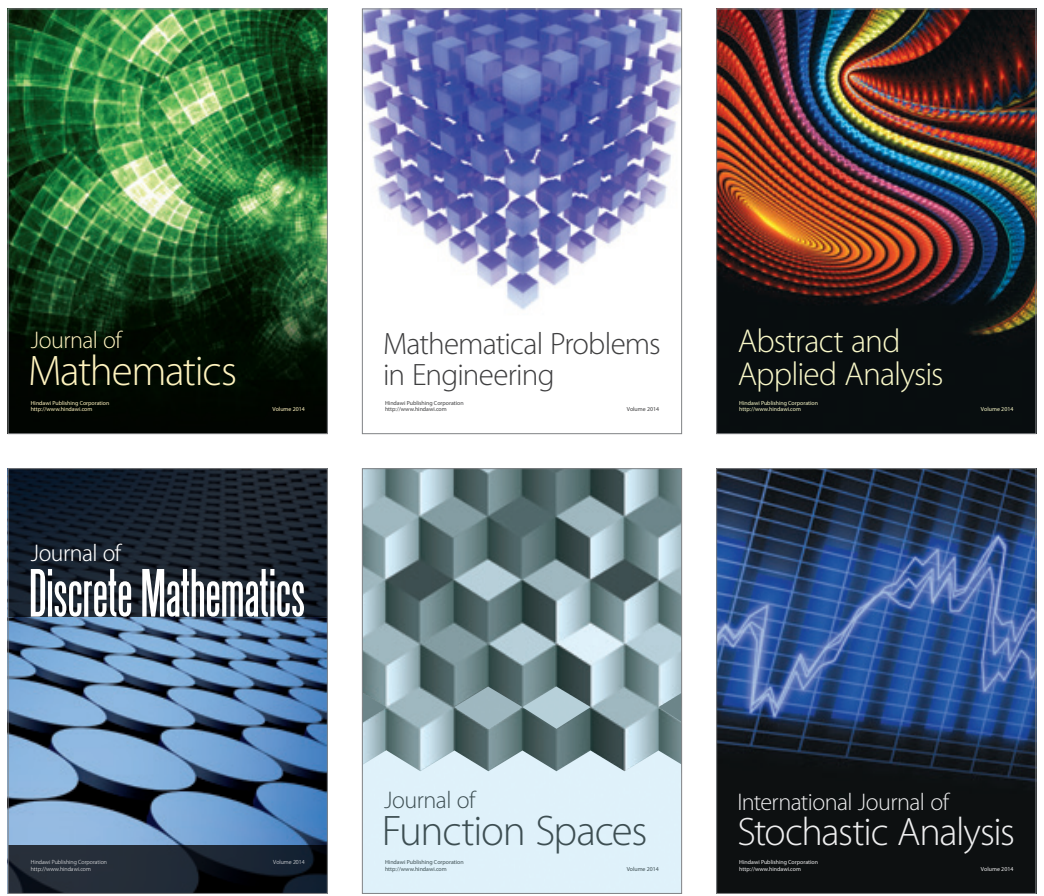

Journal of

Function Spaces

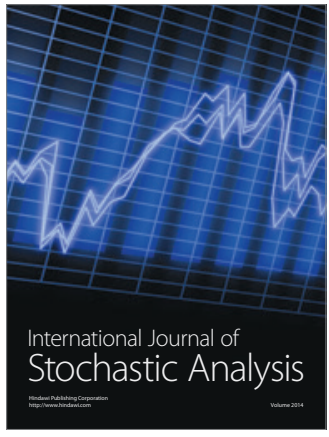

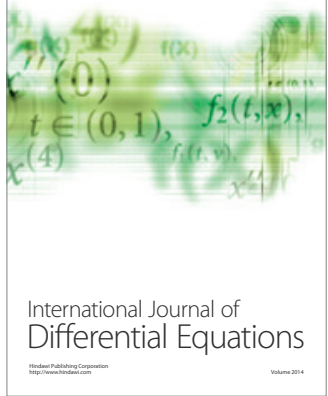
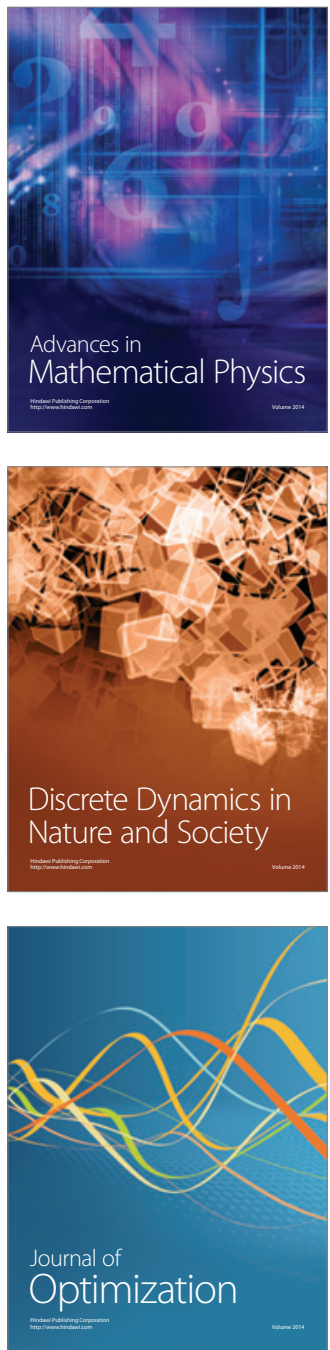